\title{
HÁBITOS ALIMENTARES, DE ATIVIDADE FÍSICA E ESTADO NUTRICIONAL DE CUIDADORES DE CRIANÇAS E ADOLESCENTES COM EXCESSO DE PESO*
}

Larissa Soares Mariz', Carla Campos Muniz Medeiros², Bertha Cruz Enders³, Ana Luisa Brandão de Carvalho Lira ${ }^{3}$, Allyne Fortes Vitor ${ }^{3}$, Caroline Evelin Nascimento Kluczynik Vieira ${ }^{1}$

'Enfermeira. Doutoranda Enfermagem. Universidade Federal do Rio Grande do Norte. Natal-RN-Brasil.

${ }^{2}$ Médica. Doutora Saúde da Criança e do Adolescente. Universidade Estadual da Paraíba. Campina Grande-PB-Brasil.

${ }^{3}$ Enfermeira. Doutora Enfermagem. Universidade Federal do Rio Grande do Norte. Natal-RN-Brasil.

RESUMO: O objetivo deste estudo foi analisar o estado nutricional e hábitos alimentares e de atividade física de cuidadores de crianças e adolescentes com excesso de peso em acompanhamento multidisciplinar. Estudo exploratório, descritivo, transversal e abordagem quantitativa com amostra de 109 cuidadores de crianças e adolescentes em atendimento no Centro de Obesidade Infantil, Campina Grande, Paraíba, Brasil. Aplicou-se questionário estruturado entre fevereiro e abril de 2011. Os resultados evidenciaram 74,3\% dos responsáveis com excesso de peso. A prática de atividade física foi considerada, em sua maioria, de forma imprópria e a alimentação classificada como regular. O hábito de comer frituras, biscoito e massas foi considerado ruim. Percebeu-se que os hábitos alimentares e de atividade física foram identificados como inadequados. O estado nutricional e as práticas alimentares e de atividade física podem refletir no estado nutricional das crianças e adolescentes em tratamento, por isso sugere-se que o atendimento de enfermagem envolva cuidadores e familiares.

DESCRITORES: Sobrepeso; Obesidade; Criança; Adolescente; Enfermagem.

\section{DIETARY HABITS, PHYSICAL EXERCISE AND NU- TRITIONAL STATUS OF CAREGIVERS OF OVER- WEIGHT CHILDREN AND ADOLESCENTS}

\begin{abstract}
The objective of this study was to assess the nutritional status and dietary and physical exercise habits of caregivers of overweight children and adolescents in multidisciplinary care. An exploratory, descriptive, crosssectional and quantitative study with a sample of 109 caregivers of children and adolescents assisted in the Center for Childhood Obesity, Campina Grande, Paraíba, Brazil. A structured questionnaire was applied from February to April 2011. The results showed that $74.3 \%$ of caregivers were overweight. The physical activity was considered mostly inappropriately and diet was classified as regular. The habit of eating fried foods, biscuits and pasta was considered bad. It was noted that the dietary and physical activity habits were inappropriate. The nutritional status and dietary and physical exercise practices may reflect in the nutritional status of children and adolescents in treatment, suggesting the inclusion of caregivers and family members in the nursing care.
\end{abstract}

DESCRIPTORS: Overweight; Obesity; Child; Adolescent; Nursing.

\section{HÁBITOS ALIMENTARES, DE ACTIVIDAD FÍSICA Y ESTADO NUTRICIONAL DE CUIDADORES DE NIÑOS Y ADOLESCENTES CON EXCESO DE PESO}

RESUMEN: El objetivo de este estudio fue analizar el estado nutricional y hábitos alimentares y de actividad física de cuidadores de niños y adolescentes con exceso de peso en acompañamiento multidisciplinar. Estudio exploratorio, descriptivo, transversal y de abordaje cuantitativo con muestra de 109 cuidadores de niños e adolescentes en atendimiento en el Centro de Obesidad Infantil, Campina Grande, Paraíba, Brasil. Se aplicó cuestionario estructurado entre febrero y abril de 2011. Los resultados evidenciaron $74,3 \%$ de los responsables con exceso de peso. La práctica de actividad física fue considerada, en mayoría, de forma impropia y la alimentación clasificada como regular. El hábito de comer frituras, biscocho y pasta fue considerado malo. Se percibió que los hábitos alimentares y de actividad física fueron identificados como inadecuados. El estado nutricional y las prácticas alimentares y de actividad física puenden reflectar en el estado nutricional de los niños y adolescentes en tratamiento, por eso se sugiere que el atendimiento de enfermería involucre cuidadores y familiares. DESCRIPTORES: Sobrepeso; Obesidad; Niño; Adolescente; Enfermería.

\footnotetext{
*Artigo extraído da Dissertação de Mestrado intitulada "Fatores de risco cardiovascular e condições associadas ao seguimento de crianças e adolescentes obesos ou com sobrepeso em um centro de referência". Universidade de Pernambuco/Universidade Estadual da Paraíba, 2011.
}

\section{Autor Correspondente:}

Caroline Evelin Nascimento Kluczynik Vieira

Universidade Federal do Rio Grande do Norte

Av. Senador Salgado Filho, 3000 - 59078-900 - Natal-RN-Brasil

E-mail: carolinekluczynik@gmail.com
Recebido: 01/02/2014

Finalizado: 21/10/2014 


\section{INTRODUÇÃO}

Dentre os problemas de saúde que acometem crianças e adolescentes está o excesso de peso. De origem multifatorial, pode ser caracterizado pelo acúmulo excessivo de tecido adiposo relacionado ao desequilíbrio entre a ingestão e o gasto energético ${ }^{(1)}$ e pode estar associado a outros agravos, tais como: doenças cardiovasculares, hipertensão arterial, diabetes, alguns tipos de neoplasias e doenças mentais, como depressão e ansiedade ${ }^{(2)}$.

As causas da obesidade são passíveis de prevenção, no entanto, é o agravo em maior evolução no mundo. No Brasil, segundo dados da Pesquisa Nacional de Saúde do Escolar, o sobrepeso está presente em 16\% dos adolescentes entre 13 e 15 anos e a obesidade, em 7,2\% ${ }^{(3)}$. Nas crianças, identificaram-se índices de 33,5\% de excesso de peso. Dados dos últimos 20 anos indicam que o percentual de meninos acima do peso mais do que dobrou e, nas meninas, a taxa quase triplicou ${ }^{(4)}$.

A fim de oferecer subsídio para a atenção integral à família com excesso de peso, em 2002 o Ministério da Saúde lançou o manual do crescimento e desenvolvimento infantil, com o objetivo de melhorar e monitorar o estado nutricional, entre os quais: baixo peso e excesso de peso $^{(5)}$. Somado a isso, em 2007, estabeleceuse o Programa de Saúde na Escola com ações em saúde previstas para crianças e adolescentes desenvolvidas articuladamente com a rede de educação pública básica, compreendendo, entre outras atividades a avaliação nutricional(6).

Portanto, percebe-se a preocupação e investimento do Ministério da Saúde do Brasil, na prevenção e acompanhamento de crianças e adolescentes com desvio nutricional. Essa crescente prevalência do excesso de peso entre os mais jovens está relacionada, principalmente, às atividades de lazer sedentárias, práticas alimentares inadequadas e fatores genéticos ${ }^{(1)}$.

Cabe neste ponto destacar a influência da família no estado nutricional das crianças e adolescentes, pois está mais relacionada ao compartilhamento de hábitos que à herança genética. Indivíduos da mesma família compartilham fatores ideológicos e culturais que influenciam a formação do hábito alimentar, a percepção de fome, apetite e saciedade ${ }^{(7)}$.
Um estudo apontou provável influência do componente familiar sobre o risco para obesidade infanto-juvenil, uma vez que a criança depende das escolhas de seus pais e familiares com relação à compra, modo de preparo dos alimentos, hábitos alimentares e incentivos à prática de atividade física ${ }^{(8)}$.

Assim, acredita-se ser relevante identificar a influência familiar sobre o estado nutricional dos mais jovens, com vistas a identificar determinantes do excesso de peso. Fato este que pode favorecer o trabalho do enfermeiro, uma vez que se trata de um profissional que faz parte da equipe multidisciplinar na prevenção e tratamento do excesso de peso na população infanto-juvenil. Ressalta-se que assistir essa clientela significa não apenas enumerar condutas, mas entender o seu contexto social e familiar(2).

Esta pesquisa justifica-se por abordar um tema atual, que está inserido nas prioridades das políticas públicas do país, especialmente as relacionadas à Política de Promoção da Saúde. Nesse contexto, entende-se como promoção da saúde a ação que ultrapassa o estímulo às práticas saudáveis e atividades oferecidas pelos serviços de saúde, e promovem a inclusão de toda a sociedade no processo saúde/doença. Com isso, espera-se a corresponsabilização das famílias e dos profissionais de saúde para transformação do meio em que estão inseridas essas crianças e suas necessidades particulares de saúde ${ }^{(9)}$.

Desse modo, por meio do presente estudo espera-se oferecer informações que possam aprimorar as ações de enfermagem para essa clientela na promoção da saúde, bem como provocar reflexões sobre o papel do enfermeiro no processo de organizar e planejar sua assistência, com olhar mais abrangente ao considerar a influência do componente familiar no desfecho do excesso de peso em crianças e adolescentes.

Partindo desse pressuposto, objetivou-se analisar o estado nutricional e hábitos alimentares e de atividade física dos cuidadores de crianças e adolescentes com excesso de peso em tratamento multidisciplinar.

\section{MÉTODO}

Trata-se de um estudo exploratório, descritivo, com delineamento transversal e análise quantitativa, realizado em um serviço de saúde 
situado em cidade do interior do estado da Paraíba, referência no tratamento de crianças e adolescentes com excesso de peso, o Centro de Obesidade Infantil (COI). Esta pesquisa faz parte de um estudo, realizado em Campina Grande/ Paraíba, no período de novembro a abril 2011, em que se acompanhou por um ano o tratamento interdisciplinar de crianças e adolescentes com excesso de peso cadastrado no COI, que incluiu investigar os hábitos dos responsáveis.

Foram considerados como responsáveis àqueles que tinham mais de 18 anos, que conduziam frequentemente a criança/ adolescente ao atendimento no $\mathrm{COI}$ ou que se responsabilizavam pelo paciente na maior parte do tempo em casa. Esses responsáveis responderam um questionário proposto na pesquisa. Conforme consta no Estatuto da Criança e do Adolescente, considera-se criança o indivíduo com menos de 11 e adolescente o indivíduo entre os 12 e 18 anos $^{(10)}$.

O processo de amostragem ocorreu por método acidental e a amostra foi composta por 109 responsáveis por crianças e adolescentes com excesso de peso, entre três e 19 anos. Os responsáveis foram recrutados mediantes cadastro no COI. Aplicou-se um questionário estruturado aos responsáveis pelas crianças e adolescentes abordando o estado nutricional e hábitos dos adultos. O instrumento abordou condutas e mudanças adquiridas na família após o ingresso do paciente no COI.

As variáveis do estudo foram: Índice de Massa Corpórea (IMC); horas por dia de atividade física; hábito de tomar café da manhã; concepção dos acompanhantes sobre a classificação da alimentação como ótima, boa, regular ou ruim; disponibilidade de mudança da prática de atividade física e alimentar no auxílio ao tratamento da criança/adolescente; mudanças nos hábitos alimentares em casa após a inserção no tratamento do excesso de peso e perfil alimentar.

Depois de responder o questionário foram coletados os dados antropométricos (peso e estatura), aferidos por dois examinadores separadamente, sendo considerado o valor médio das duas aferições. Para obtenção do peso utilizou-se uma balança digital tipo plataforma, da marca Welmy ${ }^{\circledR}$, com capacidade para 150 $\mathrm{kg}$ e precisão de $0,1 \mathrm{~kg}$. A altura foi aferida através de um estadiômetro da marca Tonelli ${ }^{\circledR}$, com precisão de 0,1 cm. Durante a aferição, o indivíduo vestia roupas leves.

Para a classificação do estado nutricional foi calculado o IMC conforme as recomendações do CDC (2000), que define as seguintes categorias: obesidade grave (IMC $\geq$ percentil 97 ), obeso (percentil $95 \geq \mathrm{IMC}<97$ ) e com sobrepeso $(85 \geq \mathrm{IMC}<95)^{(11)}$.

A prática de atividade física foi mensurada pela quantidade de horas de atividade aeróbica durante a semana (atividades físicas regulares, competitivas, individuais ou coletivas). Considerou-se como sedentário o hábito de não praticar o mínimo de 150 minutos durante a semana $^{(12)}$.

A frequência alimentar dos responsáveis foi classificada como recomendada, aceitável e ruim. Foi considerado como recomendado os alimentos protetores e seu consumo de cinco ou mais dias por semana; aceitável, de quatro dias por semana e ruim quando abaixo de quarto dias. Para os alimentos de risco foi considerado recomendado o consumo de zero a uma vez por semana; aceitável, duas vezes e ruim, três ou mais vezes por semana ${ }^{(13)}$.

Os dados foram digitados em duplicata em planilha eletrônica. Utilizou-se o software SPSS versão 17.0 para análise descritiva mediante frequência absoluta e relativa das variáveis estudadas.

O protocolo do estudo foi previamente analisado e aprovado pelo Comitê de Ética e Pesquisa da Universidade Estadual da Paraíba sob o $\mathrm{n}^{\mathrm{o}}$ 0040.0.133.000-08, de acordo com a Resolução 196/96 do Conselho Nacional de Saúde, em vigor na época do estudo. Os responsáveis firmaram o compromisso através da assinatura do Termo de Consentimento Livre e Esclarecido.

\section{RESULTADOS}

De acordo com os dados da pesquisa os 109 responsáveis, cuidadores dos pacientes do ambulatório, eram predominantemente as mães. Em relação às condições econômicas a maior parte tinha renda familiar entre um e dois salários mínimos. Da população estudada, $64,2 \%$ sobreviviam com dois ou menos salários (Tabela 1). 
Tabela 1 - Características sociodemográficas dos responsáveis por crianças e adolescentes cadastrados no Centro de Obesidade Infantil. Campina Grande-PBBrasil, 2010-2011 ( $n=109)$

\begin{tabular}{lcc}
\hline Responsáveis & $\mathbf{n}$ & $\mathbf{\%}$ \\
\hline Mãe & 89 & 81,6 \\
\hline Pai & 06 & 5,5 \\
\hline Outros & 11 & 10,1 \\
\hline Não sei & 03 & 2,8 \\
\hline Renda familiar $\left(\mathrm{SM}^{*}\right)$ & & \\
\hline $1 / 4$ a $1 / 2$ & 07 & 6,4 \\
\hline $1 / 2$ a 1 & 20 & 18,3 \\
\hline 1 a 2 & 43 & 39,5 \\
\hline 2 a 5 & 34 & 31,2 \\
\hline > 5 & 04 & 3,7 \\
\hline Não soube informar & 01 & 0,9 \\
\hline
\end{tabular}

† Salário Mínimo em 2011. Valor de R\$545,00.

As Tabelas 2 e 3 refletem os hábitos e perfil nutricional dos responsáveis pelos pacientes atendidos no ambulatório. A maior parte apresentou IMC nas classificações de sobrepeso e obesidade/obesidade grave. Com relação à prática de atividade física, a maioria não fazia nenhuma atividade e, dentre os que realizavam alguma atividade, tinham uma frequência dessa prática de 1 a 2 vezes por semana.

Sobre a alimentação, os sujeitos a autoclassificaram como boa. A maior parte destes afirmou ter mudado o hábito alimentar da família junto à criança ou adolescente tratado. Contudo, ainda $1 / 4$ deles afirmou ter dificuldades para mudar a alimentação e a prática de atividade física.

Os relatos sobre comer frituras, biscoito e massas foram considerados predominantemente como ruins e os refrigerantes estavam presentes em metade da população, sendo consumidos de forma aceitável. Por outro lado, identificou-se um bom consumo de frutas e verduras (Tabela 3).
Tabela 2 - Estado nutricional e hábitos dos responsáveis por crianças e adolescentes cadastrados no Centro de Obesidade Infantil. Campina Grande-PB-Brasil, 2010$2011(n=109)$

\begin{tabular}{|c|c|c|}
\hline Índice de massa corporal & $\mathbf{n}$ & $\%$ \\
\hline Obesidade grave & 19 & 17,5 \\
\hline Obeso & 28 & 25,7 \\
\hline Sobrepeso & 36 & 33 \\
\hline Eutrófico & 26 & 23,8 \\
\hline \multicolumn{3}{|l|}{ Atividade física (horas/dia) } \\
\hline Nenhuma & 83 & 76,2 \\
\hline 1 a 2 & 21 & 19,3 \\
\hline 3 a 4 & 01 & 0,9 \\
\hline 5 a 6 & 00 & 0 \\
\hline$>6$ & 02 & 1,8 \\
\hline Não sei & 02 & 1,8 \\
\hline \multicolumn{3}{|c|}{ Hábito tomar café da manhã } \\
\hline Nunca & 02 & 1,8 \\
\hline Raramente & 02 & 1,8 \\
\hline Às vezes & 11 & 10,1 \\
\hline Sempre & 92 & 84,5 \\
\hline Não soube informar & 02 & 1,8 \\
\hline \multicolumn{3}{|c|}{ Como classifica sua alimentação } \\
\hline Ótima & 08 & 7,3 \\
\hline Boa & 52 & 47,8 \\
\hline Regular & 33 & 30,3 \\
\hline Ruim & 14 & 12,8 \\
\hline Não informou & 02 & 1,8 \\
\hline \multicolumn{3}{|c|}{ Mudaria a alimentação para ajudar o paciente } \\
\hline Sim, sem dificuldade & 79 & 72,5 \\
\hline Sim, com dificuldade & 27 & 24,8 \\
\hline Não & 01 & 0,9 \\
\hline Não soube informar & 02 & 1,8 \\
\hline
\end{tabular}

Mudaria o hábito de atividade física para ajudar o paciente

\begin{tabular}{lcc}
\hline Sim, sem dificuldade & 74 & 67,9 \\
\hline Sim, com dificuldade & 31 & 28,5 \\
\hline Não & 02 & 1,8 \\
\hline Não soube informar & 02 & 1,8 \\
\hline \multicolumn{2}{l}{ Houve mudanças nos hábitos de casa? } \\
\hline Não & 17 & 15,5 \\
\hline Sim & 66 & 60,6 \\
\hline Pouco & 26 & 23,9 \\
\hline
\end{tabular}


Tabela 3 - Perfil alimentar dos responsáveis por crianças e adolescentes cadastrados no Centro de Obesidade Infantil. Campina Grande-PB-Brasil, 2010-2011 ( $\mathrm{n=109)}$

\begin{tabular}{|c|c|c|}
\hline Frutas & $n$ & $\%$ \\
\hline Recomendável & 57 & 52,3 \\
\hline Aceitável & 04 & 3,7 \\
\hline Ruim & 48 & 44 \\
\hline \multicolumn{3}{|l|}{ Verduras } \\
\hline Recomendável & 69 & 63,3 \\
\hline Aceitável & 05 & 4,6 \\
\hline Ruim & 35 & 32,1 \\
\hline \multicolumn{3}{|l|}{ Fritura } \\
\hline Recomendável & 23 & 21,1 \\
\hline Aceitável & 40 & 36,7 \\
\hline Ruim & 46 & 42,2 \\
\hline \multicolumn{3}{|l|}{ Doces } \\
\hline Recomendável & 50 & 45,9 \\
\hline Aceitável & 30 & 27,5 \\
\hline Ruim & 29 & 26,6 \\
\hline \multicolumn{3}{|l|}{ Biscoito } \\
\hline Recomendável & 39 & 35,8 \\
\hline Aceitável & 21 & 19,2 \\
\hline Ruim & 49 & 45 \\
\hline \multicolumn{3}{|l|}{ Massas } \\
\hline Recomendável & 16 & 14,7 \\
\hline Aceitável & 30 & 27,5 \\
\hline Ruim & 63 & 57,8 \\
\hline \multicolumn{3}{|l|}{ Refrigerante } \\
\hline Recomendável & 33 & 30,3 \\
\hline Aceitável & 58 & 53,2 \\
\hline Ruim & 18 & 16,5 \\
\hline
\end{tabular}


Quanto ao estado nutricional dos responsáveis verificou-se que mais da metade tinha excesso de peso, sendo $33 \%$ com sobrepeso e $25,7 \%$ obesos. Uma revisão sistemática afirmou que as causas e fatores desencadeantes da obesidade infantil são diversos, dentre eles estão a hereditariedade e o ambiente familiar, os quais foram denominados de contexto potencial envolvido com a obesidade infanti $i^{(17)}$. Outro estudo concluiu que o peso dos pais influencia seus filhos, por exemplo, mães obesas exercem maior controle no consumo dos filhos, deste modo o IMC das crianças está associado ao dos pais ${ }^{(15)}$.

Por isso, sugere-se que o enfermeiro, como promotor da saúde e agente ativo no tratamento do excesso de peso, interaja com os responsáveis a fim de compreender seu conhecimento acerca das consequências desse problema em crianças e adolescentes, para assim identificar a visão sobre o estado nutricional dos filhos, utilizando para isso um olhar qualificado. Sugere-se também que seja esclarecido que o tratamento é um processo lento e de envolvimento de toda família ${ }^{(16)}$.

Os responsáveis devem estar atentos às atividades do dia a dia, como as compras do supermercado, mostrar e incentivar a compra de verduras, frutas e legumes, oferecer alimentos variados e acompanhar o peso e a altura da criança/adolescente periodicamente ${ }^{(7)}$.

Ao enfermeiro, como parte da equipe multiprofissional no atendimento a essa clientela, sugere-se: reservar um espaço de tempo para esclarecimento de dúvidas dos pacientes ou dos responsáveis; ouvir os medos, ansiedades e objetivos de ambos; fornecer orientação para consumo de alimentos saudáveis e menos dispendiosos, compatível ao alcance econômico da família; e estimular a prática de atividade física para o paciente e família( ${ }^{(18)}$.

\section{CONCLUSÃO}

Os cuidadores de crianças e adolescentes com excesso de peso, que receberam acompanhamento por serviço de referência, apresentaram em sua maioria estado nutricional de excesso de peso, afirmaram consumir frequentemente alimentos não saudáveis e praticar pouca atividade física.

Portanto, identificou-se nos cuidadores um perfil nutricional e hábitos que poderiam influenciar negativamente no tratamento das crianças e adolescentes com excesso de peso. Sugere-se que os enfermeiros considerem ações de saúde que envolvam a família, de forma que o estímulo aos hábitos saudáveis e o acompanhamento do estado nutricional envolvam não apenas a criança e adolescente com excesso de peso.

Como limitações do estudo tem-se o fato de ser transversal, o que impossibilitou a inferência de causalidade. Portanto, sugere-se o desenvolvimento de novos estudos, através do acompanhamento por maior período, a fim de esclarecer as multicausalidades da obesidade, como análise da influência genética, comportamental e socioeconômica.

\section{REFERÊNCIAS}

1. Hernandes F, Valentini MP. Obesidade: causas e consequências em crianças e adolescentes. Revista da Faculdade de Educação Física da UNICAMP. 2010;8(3). [acesso em: 23 ago 2014]. Disponível em: http://fefnet178.fef.unicamp.br/ojs/index.php/fef/ article/view/576/379

2. Leal VS, Lira PIC, Oliveira JS, Menezes RCE, Sequeira LAS, Arruda Neto MA, et al. Excesso de peso em crianças e adolescentes no Estado de Pernambuco, Brasil: prevalência e determinantes. Cad. saude publica. [Internet] 2012; 28(6). [acesso em: 17 mai 2014] Disponível em: http://dx.doi.org/10.1590/S0102311X2008000500006

3. Ministério da Saúde (BR). Pesquisa Nacional de Saúde do Escolar (PENSE). Rio de Janeiro: IBGE; 2009.

4. Ministério do Planejamento, Orçamento e Gestão (BR). Pesquisa de Orçamentos Familiares (POF) 2008-2009: despesas, rendimentos e condições de vida. Rio de Janeiro: IBGE; 2010.

5. Ministério da Saúde (BR). Secretaria de Políticas de Saúde. Saúde da criança: acompanhamento do crescimento e desenvolvimento infantil. Brasília: Ministério da Saúde; 2002.

6. Ministério da Saúde (BR). Política Nacional de Alimentação e Nutrição. Série B. Textos Básicos de Saúde. Brasília: Ministério da Saúde; 2008.

7. Camargo APPM, Barros FAA, Antonio MARGM, Giglio JS. A não percepção da obesidade pode ser um obstáculo no papel das mães de cuidar de seus filhos. Ciênc. saúde coletiva [Internet]. 2013;18(2). [acesso em: 06 jan 2014]. Disponível em: http://dx.doi. org/10.1590/S1413-81232013000200004 
8. Silveira A, Jansen A, Norton R, Silva, G, Whyte P. Efeito do atendimento multidisciplinar na modificação dos hábitos alimentares e antropometria de crianças e adolescentes com excesso de peso. Rev Med Minas Gerais [Internet]. 2010;20 (3). [acesso em: 23 ago 2014]. Disponível em: http://www.medicina.ufmg.br/ rmmg/index.php/rmmg/article/viewFile/265/248

9. Pilar ACA, Andrade M. Promoção da Saúde: uma reflexão sobre o papel do enfermeiro. Informe-se em promoção da saúde. 2011;7(1):5-8.

10. Brasil. Lei n. 8.069, de 13 de julho de 1990. Dispõe sobre o Estatuto da Criança e do Adolescente. Diário Oficial da República Federativa do Brasil, Brasília, 16 jul. 1990, p.13563.

11. Centers for Diseases Control and Prevention (CDC). Table for calculated body mass index values for selected heghts and weights for ages 2 to 20 years. Estados Unidos: National Center for Health Statistc and National Center for Chronic Disease Prevention and Health Promotion; c2000.

12. WHO - World Health Organization. Obesity and overweight: Global strategy on diet, physical activity and healthy [Internet]. Geneva; 2009. [acesso em: 16 nov 2013]. Disponível em: www.who.int/ dietphysicalactivity/media/en/gsfs_obesity.pdf

13. Ministério da Saúde (BR). Vigilância de Fatores de Risco e Proteção para Doenças Crônicas por Inquérito Telefônico - VIGITEL [Internet]. Brasília; 2010 [acesso em: 02 set 2013]. Disponível: http://portal.saude.gov. br/portal/arquivos/pdf/vigitel_180411.pdf

14. Koehnlein EA, Salado GA, Yamada AN. Adesão à reeducação alimentar para perda de peso: determinantes, resultados e a percepção do paciente. Rev Bras Nutr Clin. 2008;23(1) [acesso em: 17 mai 2014]. Disponível: http://saudeemovimento.net.br/ wp-content/uploads/bsk-pdf-manager/10_2014-07-05. PDF

15. Costa FS, Daisy LDP, Friedman R. Caregivers attitudes and practices: influence on childhood body weight. J. biosoc. sci. [Internet] 2011;43. [acesso em: 23 ago 2014]. Disponível em: http://diabetesendocrinologia. org.br/pdfs_abril_11/Costa+et+al+ JBS+2011.pdf

16. Souza MHN, Silveira GS, Pinto AFS, Sodré VRD, Ghelman LG. Avaliação do estado nutricional e da saúde de crianças e adolescentes na prática assistencial do enfermeiro. Cogitare enferm. [Internet]. 2013;18(1). [acesso em: 17 mai 2014] Disponível: http://ojs.c3sl.ufpr.br/ojs/index.php/cogitare/article/ viewFile/28926/20010

17. Rocha RM, Soares RS, Silva JLL, Lima JL, Valente C, Soraia G. Prevalence of obesity in children introduced in the elementary school: systematic review. Rev enferm UFPE on line. 2013;7(3):849-56.
18. Sarti TD, Campos CEA, Zandonade E, Ruschi GEC, Maciel ELN. Avaliação das ações de planejamento em saúde empreendidas por equipes de saúde da família. Cad. saude publica. [Internet]. 2012;28(3). [acesso em: 10 mar 2014] Disponível em: http://dx.doi.org/10.1590/ S0102-311X2012000300014 